\title{
Chikungunya Virus Infection
}

\author{
Fabrice Simon • Emilie Javelle • Manuela Oliver • \\ Isabelle Leparc-Goffart • Catherine Marimoutou
}

Published online: 6 April 2011

(C) The Author(s) 2011. This article is published with open access at Springerlink.com

\begin{abstract}
Chikungunya virus (CHIKV) is an alphavirus transmitted by mosquitoes, mostly Aedes aegypti and Aedes albopictus. After half a century of focal outbreaks of acute febrile polyarthralgia in Africa and Asia, the disease unexpectedly spread in the past decade with large outbreaks in Africa and around the Indian Ocean and rare autochthonous transmission in temperate areas. This emergence brought new insights on its pathogenesis, notably the role of the A226V mutation that improved CHIKV fitness in Ae. albopictus and the possible CHIKV persistence in deep
\end{abstract}

F. Simon $(\bowtie) \cdot$ E. Javelle

Department of Infectious Diseases and Tropical Medicine,

Laveran Military Teaching Hospital,

Marseille, France

e-mail: simon-f@wanadoo.fr

E. Javelle

e-mail: emilie.javelle@gmail.com

M. Oliver

Laboratory of Medical Biochemistry,

Laveran Military Teaching Hospital,

Marseille, France

e-mail: manuelaoliver@bbox.fr

I. Leparc-Goffart

Department of Tropical Virology,

Associate National Reference Center for Arboviruses,

Institute for Biomedical Research of the French Army,

Marseille, France

e-mail: leparcgoffart@gmail.com

C. Marimoutou

Clinical Research Unit, LAVERAN Military Teaching Hospital,

Marseille, France

e-mail: marimoutouc@aol.com

C. Marimoutou

LERTIM, Université de la Méditerranée,

Marseille, France tissue sanctuaries for months after infection. Massive outbreaks also revealed new aspects of the acute stage: the high number of symptomatic cases, unexpected complications, mother-to-child transmission, and low lethality in debilitated patients. The follow-up of patients in epidemic areas has identified frequent, long-lasting, rheumatic disorders, including rare inflammatory joint destruction, and common chronic mood changes associated with quality-of-life impairment. Thus, the globalization of CHIKV exposes countries with Aedes mosquitoes both to brutal outbreaks of acute incapacitating episodes and endemic long-lasting disorders.

Keywords Chikungunya $\cdot$ Alphavirus $\cdot$ Rheumatism . Emergence $\cdot$ Chronicity

\section{Introduction}

The chikungunya virus (CHIKV) is an arthropod-borne virus transmitted by Aedes mosquitoes that is mostly responsible for acute and chronic articular manifestations [1•]. It has become a new, unexpected public health problem in many tropical African and Asian countries within the past decade, and is now considered a real threat to temperate areas colonized by Aedes spp, such as Europe and the Americas [1•]. First described in Africa (Tanzania) in 1954 and then identified in Asia, it was responsible for widespread outbreaks on these two continents from the 1960 s to the 1980 s, before a period of relative quiescence over the following 20 years. In 2000, a huge outbreak in the Congo [2] announced a resurgence of the disease, and its global emergence really started in 2004. A major outbreak first spread from Eastern Africa, in Kenya, to most islands in the Indian Ocean in 2005-2006 [3], notably infecting 
one third of the 770,000 inhabitants of Reunion Island [4]. CHIKV spread progressively toward Asia, affecting India since 2006 [5] and successively most countries of the region. Hundreds of intercontinental travelers were also infected while staying in epidemic countries $[1 \cdot, 6,7]$. Unexpectedly, in 2007, Italy underwent a small autochthonous outbreak that developed from a single viremic patient returning from India [8]. In September 2010, in southeastern France, two cases of autochthonous transmission of chikungunya fever were described (Leparc-Goffart, personal communication) and focal transmission has been since observed in Southern China, Arabic peninsula and New Caledonia in Pacific Ocean. These events confirmed the epidemic potency of this arboviral disease and definitely showed the risk for temperate or remote areas.

With this recent global emergence witnessing millions of infected patients, our knowledge of the disease significantly changed. Before 2004, it was considered a minor arboviral disease from an epidemiological and clinical point of view. Chikungunya was mostly perceived as a benign acute painful fever, and commonly mistaken for dengue fever. Its first description was limited to a brutal fever, a constant peripheral polyarthritis occasionally associated with a rash. The intensity of the pain and handicap gave the disease its name: "chikungunya" means "that which bends up" in Makonde language. Studies from the recent outbreaks described the features of the acute stage, including atypical, severe, and lethal complications, and showed the burden of the chronic stage $[4,9-11]$. This review aims to present the pathogenesis of CHIKV, its epidemiological changes, individual consequences, and future challenges.

\section{Chikungunya Virus: An Alphavirus and an Arbovirus}

\section{Virological Aspects and Pathogenesis}

CHIKV belongs to the alphavirus genus of the Togaviridae family [12]. It belongs to the Semliki Forest virus antigenic complex that also contains the O'Nyong Nyong, Mayaro, and Ross River viruses. It is an enveloped positive-strand RNA virus, with a genome of about $12 \mathrm{~kb}$. The genome is capped in $5^{\prime}$ and has a polyA tail in the $3^{\prime}$ end. It encodes four nonstructural proteins (nsP1 to nsP4) and five structural proteins (C-E3-E2-6 k-E1). Genetic analysis based on the E1 envelope glycoprotein sequences showed three distinct lineages: the West African cluster, the EastCentral and South African cluster (ECSA), and the Asian cluster [13]. It is thought that CHIKV originated in West Africa, colonized other African areas, and was secondarily introduced into Asia before the 1960s [14••]. The viral situation remained stable for five decades. Strains circulating in the Western Indian Ocean in the 2000s were related to the ESCA lineage [12]. The most significant event in CHIKV history was the appearance of an adaptive mutation, an alanine-to-valine substitution at position 226 in the E1 glycoprotein gene (E1:A226V) on an ESCA-CHIKV strain circulating on Reunion Island after September 2005. It led the mutated CHIKV to lose cholesterol dependence for growth and enhanced its infectivity, replication, and transmission by Ae. albopictus, without impairing common vectorial capability of Ae. aegypti $[15,16]$. Similar genetic events occurred independently in India, Gabon, and Cameroon, suggesting an evolutionary convergence of the virus to this mosquito and a great subsequent ability for worldwide epidemic expansion [13, 17, 18]. To date, no difference in virulence between the different strains of CHIKV has been shown in humans.

The pathophysiology of human CHIKV infection has long been a mystery. Some hypotheses were first proposed based on data from other alphaviruses. Significant progress was made after studies on chikungunya animal models and on target cells for CHIKV replication in humans. CHIKV could be detected in connective tissue (especially in epimysium), in muscle, joint, and skin fibroblasts, and even in the central nervous system (CNS) in some severe mouse infections but not in fetal or placental tissues $[19,20]$. Nevertheless, murine models are limited because the CHIKV infection in the mouse does not present all the patterns of the human disease. Labadie et al. [21••] described a promising model of CHIKV infection in Cynomolgus macaques showing clinical and pathological signs close to those observed in humans. In this model, at the early stage of the disease, the organs targeted for CHIKV replication were lymphoid tissues, liver, CNS, joints, and muscle, and the persistence of CHIKV could be found later in the lymphoid organs, liver, joints, and muscle, macrophages being the main reservoir for persistent CHIKV infection. In humans, acute CHIKV infection is characterized by a very early viremia at fever onset that can increase up to $10^{9}$ to $10^{12}$ RNA copies/mL and lasts up to 12 days [22]. CHIKV and IgM anti-CHIKV have been found in the cerebrospinal fluid of patients with acute encephalitis [20]. In vitro studies have shown that human epithelial and endothelial cells, primary fibroblasts, and monocyte-derived macrophages are susceptible to CHIKV infection, whereas activated $\mathrm{B}$ and $\mathrm{T} \mathrm{CD}^{+}$lymphocytes, monocytes, and monocyte-derived dendritic cells were refractory to $\mathrm{CHIKV}$ infection [23]. CHIKV was also detected in the biopsies of muscle, joint, and dermis of infected human patients; CHIKV antigens were mostly confined to the fibroblasts of skeletal muscle epimysium, of the joint capsule, and of the dermis $[19,20]$. When studying muscle biopsies of two patients with myositic syndrome (one present 3 months after disease onset), Ozden et al. [24] found CHIKV antigens only in human muscle progenitor cells (satellite cells). The persistence of CHIKV and/or CHIKV-encoded components 
in deep sanctuaries could play a role in chronic symptoms and biological changes in human infection, such as the slow disappearance of anti-CHIKV IgM in the serum of patients with long-lasting arthralgias [25]. Recent studies showed that the immune response in the acute stage is characterized by a rapid innate immune antiviral response with robust dendritic/ $\mathrm{NK} / \mathrm{CD}^{+} / \mathrm{CD}^{+}$activation and a paradoxically rather mild Th1/Th2 cytokine response in both recovered and chronic patients. In contrast, a prolonged antiviral immune response with intense interferon- $\alpha$ and interleukin-12 production was present only in chronic patients [26••]. These data are consistent with the hypothesis of a persistent viral challenge with a plausible direct contribution to synovial tissue damage. It also supports the use of immunomodulation with disease-modifying antirheumatic drugs for the most affected chronic patients, despite a theoretical risk of viral reactivation $[22,26 \bullet \bullet]$.

\section{Epidemiological Features}

Besides being an alphavirus, CHIKV is also an arthropodborne virus sharing the epidemiological characteristics of the conceptual group of arboviral diseases: transmission by a susceptible mosquito, possible expansion in colonized areas, and prevention using measures for vector control. Nevertheless, in terms of individual management and public health, any confusion with dengue fever could weigh heavily because of the clinical and socioeconomic consequences of CHIKV outbreaks $[1 \cdot 10]$. Two examples illustrate the strong impact of CHIKV outbreaks. In the Union of Comoros in 2005, about $80 \%$ of the CHIKV- seropositive population were hospitalized or stayed at home in bed for a mean of 6 days [27]. In India, the national burden of the CHIKV outbreak in 2006 was estimated to be 25,588 disability-adjusted life years, $69 \%$ of which were attributed to persistent arthralgia [28].

During five decades (1950-2000), only two epidemiological profiles of chikungunya fever were described (Fig. 1). The classical African profile is characterized by occasional small epidemics in rural areas similar to sylvatic yellow fever and with little impact on public health. Conversely, the classical Asian profile is responsible for urban outbreaks involving rather anthropophilic mosquitoes, mainly $A e$. aegypti as observed in dengue fever. The situation changed rapidly in Reunion Island, where the new A226V-CHIKV strain quickly became predominant and generated an explosive outbreak because of its adaptation to Ae. albopictus, a previously minor vector for CHIKV [12]. This diurnal, anthropophilic, and peridomestic mosquito was the most prevalent vector in urban areas of the island, conversely to other islands of the Indian Ocean. This outbreak swept through Reunion Island at the beginning of 2006, after favorable climatic conditions for mosquito proliferation. The brutal disorganization of the health system (eg, overload of hospital structures, interruption of blood donation and grafts) was rapidly followed by difficulties in vector control, media excitement, and social crisis [1•]. A third epidemiological profile was born, distinct from those of Africa and Asia and having a simple cycle well suited for invading certain urban areas (Fig. 1). The A226V-CHIKV is highly efficient at spreading through human populations in tropical and temperate countries
Fig. 1 Epidemiological features of chikungunya transmission

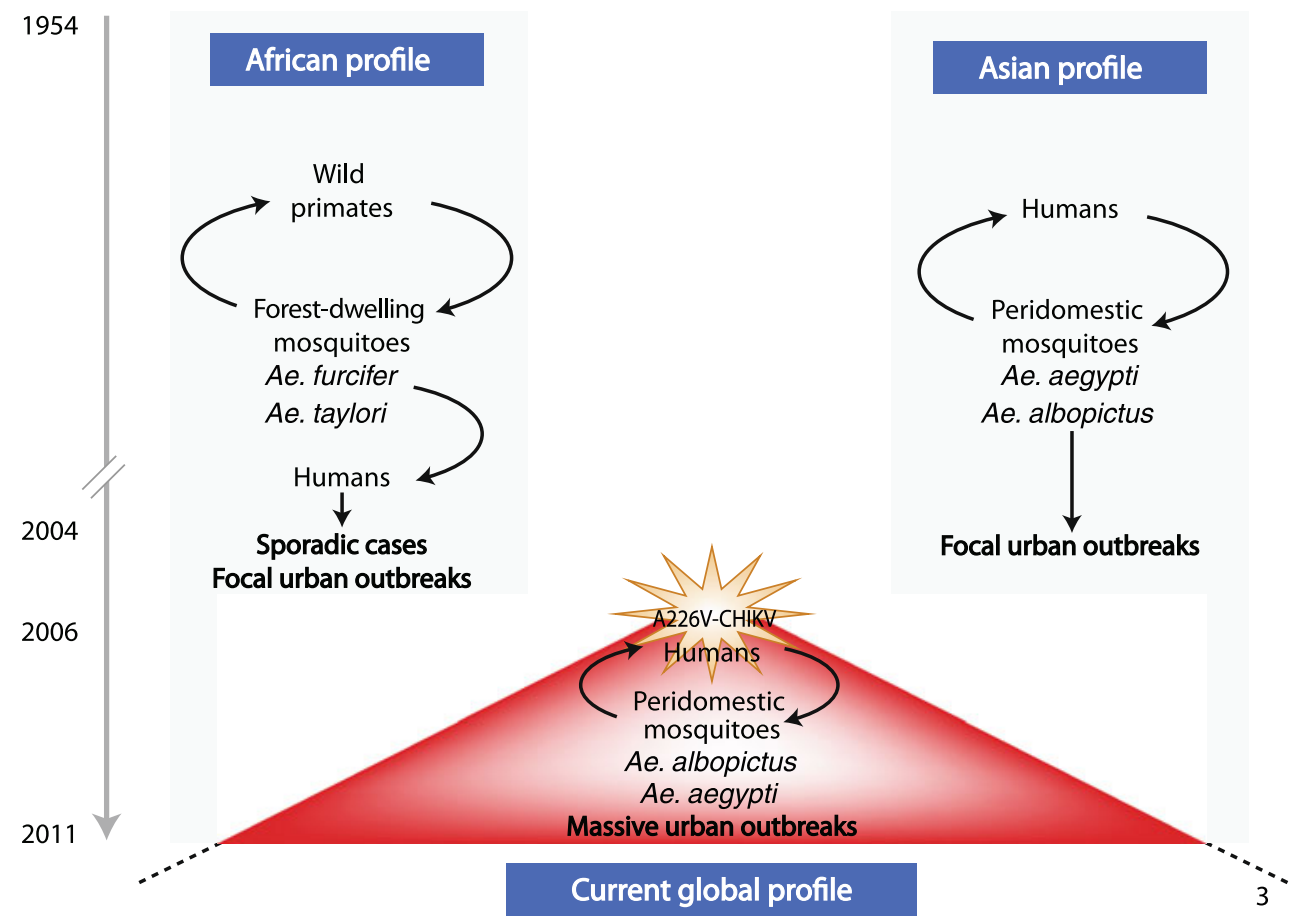


when colonized by Ae. albopictus. Within the past 6 years, this global profile has been responsible for more than 2 million cases, mostly during outbreaks in the Indian Ocean, India, and South-Eastern Asia, but also in Africa and in Europe, whereas "only" a few hundred thousand cases were reported for African and Asian profiles during the past five decades. Thus, to date, numerous countries have been concerned on three continents with outbreaks or autochthonous cases of CHIKV infection, whether mutated or not (Table 1). Hundreds of infections were recently diagnosed in returning travelers worldwide. Any CHIKV-viremic traveler arriving in an area colonized by Ae. albopictus or any other competent mosquito (eg, Ae. aegypti) can be the source of autochthonous transmission, as witnessed in Northern Italy in 2007 [8], in southeastern France in 2010 and more recently in Yemen, China and New Caledonia (Leparc-Goffart, personal communication). For this reason, CHIKV infection is a threat to large parts of the world where Aedes spp are already present or could settle under favorable climatic conditions, especially Southern Europe, the Americas, Caribbean and Pacific islands, and Australia (Table 1).

\section{Diagnosis of Chikungunya Disease}

Laboratory diagnosis relies upon the detection of the virus on early samples and/or specific anti-CHIKV IgM and IgG on blood samples.

Numerous quantitative reverse transcription-polymerase chain reactions (RT-PCR) have been developed for CHIKV detection. Commercial kits are available, sometimes with excellent sensitivity and specificity [29]. CHIKV-RNA is detected in plasma samples within the first week after symptom onset, commonly with extremely high levels of viremia [22]. RT-PCR can also be used to screen various fluids and tissues, including corneas or other graft tissues. CHIKV can also be isolated from early samples on C6/36 or Vero cell lines. This method is only performed in Biosafety Level 3 laboratories, and is therefore mainly used for epidemiological purposes and research studies.

Anti-CHIKV antibodies can be detected in patients shortly after symptom onset, usually after 5 days for IgM and only a few days later for IgG. Commercial enzyme immunoassays and immunofluorescence assays are available, but are of poor performance when expertized [30•]. Interpretation of serological results must be cautious because of 1) possible false negativity due to CHIKVinduced mixed cryoglobulinemia [25], 2) cross-reactivity with viruses of the Semliki Forest serocomplex requiring seroneutralization, and 3) long-term persistence of antiCHIKV IgM months after disease onset. Synchronous testing of a sample from the acute stage and a sample collected at least 3 weeks later is sufficient to demonstrate a recent CHIKV infection in most cases. Any doubt should lead to a request for assistance from an expert laboratory.

The good management of patients with acute CHIKV infection is essential for public health in susceptible areas with current Aedes spp activity. In these areas, most health authorities recommend prompt suspicion of imported or autochthonous cases, adequate use of diagnostic tools, isolation of suspect patients, rapid contact with the local health department, and sometimes mandatory case declaration. The final aim is to avoid epidemics spreading around the new cases.

\section{Chikungunya Infection, a Two-Stage Disease}

\section{Acute Stage}

Most patients infected with CHIKV develop acute symptoms, usually 2 to 6 days after the infective mosquito bite. In Reunion Island in 2005-2006, the prevalence of asymptomatic infections was about $5 \%$ to $10 \%$ [4]. The first symptoms start abruptly and last for about a week before spontaneous improvement. The acute stage is defined as the first 10 days after disease onset [9]. The most frequent symptoms are high fever, arthralgias, back pain, and headache [31]. Fever is usually high, and is poorly responsive to antipyretics. Illness is associated with intense fatigue, anorexia, myalgias, nausea, and vomiting in adults, and even transient confusion in elderly patients. In Reunion Island in 2006, $47.3 \%$ of adults infected considered their fatigue as significant or very significant, $6 \%$ were depressed, and $35.5 \%$ were demoralized during the acute phase [10]. The acute bilateral and symmetrical rheumatism is typically extensive and progressive within a few days [6,9]. Peripheral joints are frequently very painful and swollen, especially interphalangeal joints, wrists, and ankles. The axis and proximal joints can also become inflamed with large joint effusions in the knees and elbows. The intensity of the symptoms prompts most patients to search for efficient treatment. Unfortunately, no antiviral drug has proved effective against human CHIKV infection [32]. Thus, the treatment of the acute stage is limited to painkillers and nonsteroidal anti-inflammatory drugs (NSAIDs). Their efficacy is not complete, and adverse effects are not rare as a result of excessive selfmedication. Acetaminophen is the elective drug. Aspirin should be avoided because of the risk of bleeding. Although sometimes dramatically effective, systemic corticosteroids are not recommended because of the strong rebound effect after stopping. A transient maculopapular rash, sometimes edematous and/or pruritic, can also be observed on the face (Fig. 2a) and the trunk of half the patients [9]. Miscellaneous cutaneous and mucosal changes have been reported during the acute stage of the 


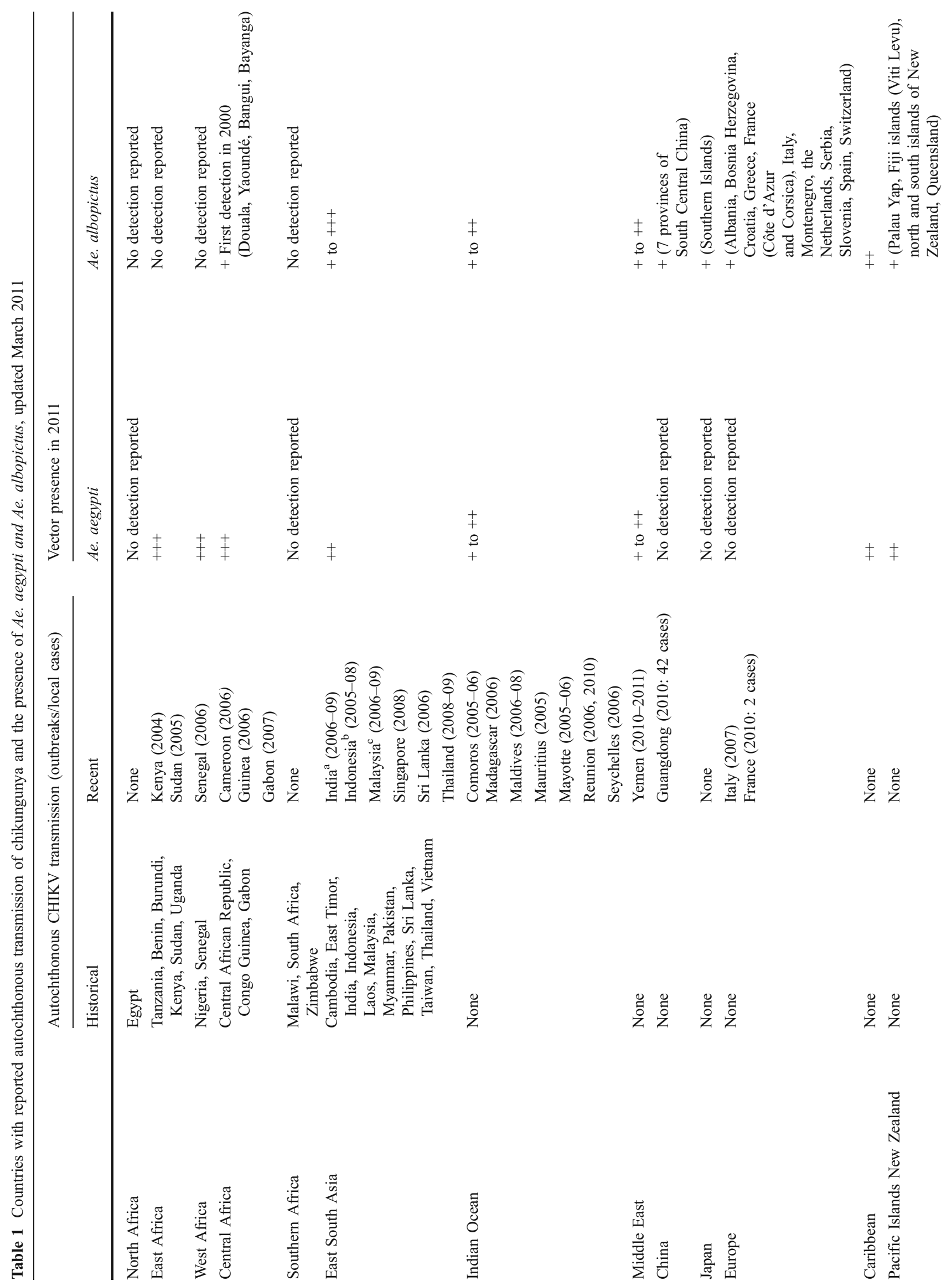




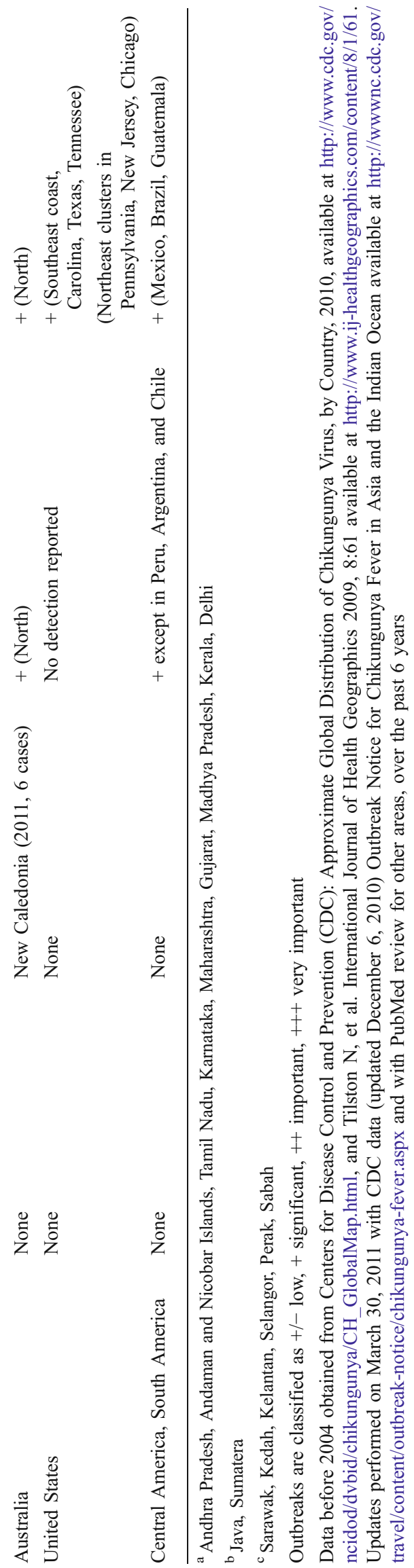

disease: photosensitivity, stomatitis, mouth ulcers, exfoliative dermatitis, vesicles, bullae, and purpura [31]. Gastrointestinal symptoms are common [11]. Initial biological changes are transient leukopenia and lymphopenia, mild thrombocytopenia, low rises in C-reactive protein, and hepatic cytolysis $[9,11,33]$. Table 2 presents some clinical and biological signs that can help to distinguish chikungunya from dengue fever. After a week of intense discomfort, pain, and incapacity, most CHIKV-infected patients experience a significant improvement of their condition. The fever drops, and the asthenia and joint pain become more acceptable. This period usually lasts for 1 to 2 weeks before a very common relapse.

Beside the typical fever-arthralgias-rash association, some clinical features and complications were recently described. Atypical features of CHIKV infection leading to hospitalization and/or death were prospectively recruited in Reunion Island, reflecting their incidence, clinical aspects, and outcome [34]. The most frequent complications involved the CNS: convulsions, meningoencephalitis, and Guillain-Barré syndrome; the direct role of CHIKV is evident in these early manifestations [35]. Other severe acute complications have been reported since 2005: myocarditis [36], fulminant hepatitis in patients with chronic liver diseases [11], pancreatitis, acute endocrine disorders [37], extensive epidermolysis [11], kidney failures, respiratory failures, and decompensation of cardiovascular diseases [34]. Although minor bleedings were observed [11], CHIKV infection is no longer considered as a viral hemorrhagic fever. CHIKV infection in children resembles that of adults, but can occasionally be complicated with neuropsychological changes, including lethal meningoencephalitis, with myocarditis or extensive epidermolysis [38].

Atypical and complicated features are exceptional $(<0.5 \%$ of cases) and more frequently observed in elderly patients and/or in the presence of underlying diseases such as hypertension, cardiovascular, or respiratory conditions [37]. During the outbreak in Reunion Island, the mortality rate was estimated to be $0.3 \%$ to $1 \%$, and increased with age, with excess deaths being observed mostly in patients over 75 years $[4,34]$.

The most surprising discovery was the mother-to-child transmission, with CHIKV neonatal infections [39]. The risk is not negligible when considering that, in Reunion Island in March 2006, about one pregnant woman in five had serological markers of recent infection [4]. In India, the risk of abortion and fetal death increased before the 22nd week of pregnancy. There was no increased risk for malformation [40]. Mother-to-child transmission were only observed in near-term deliveries for CHIKV-viremic mothers [39]. Under these conditions, half the neonates were infected. Neonatal CHIKV infection was symptomatic after 4 days in one in two cases, with fever, pain, prostration, 
a

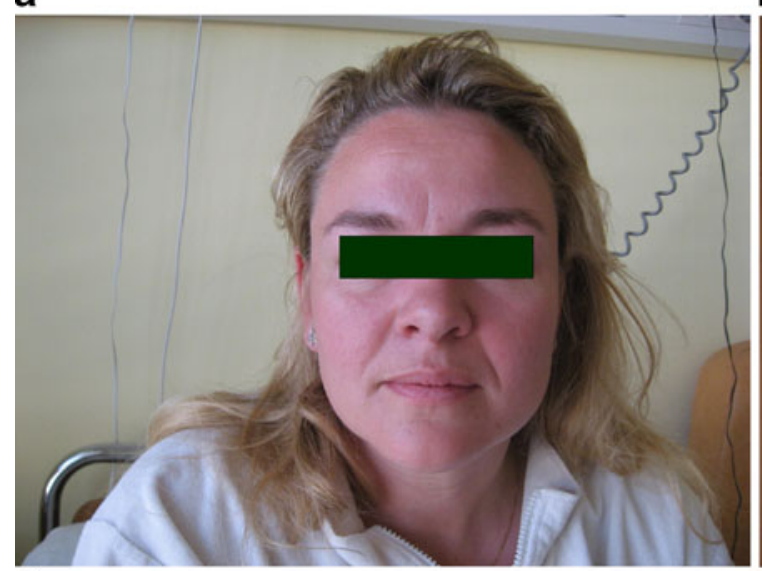

C b
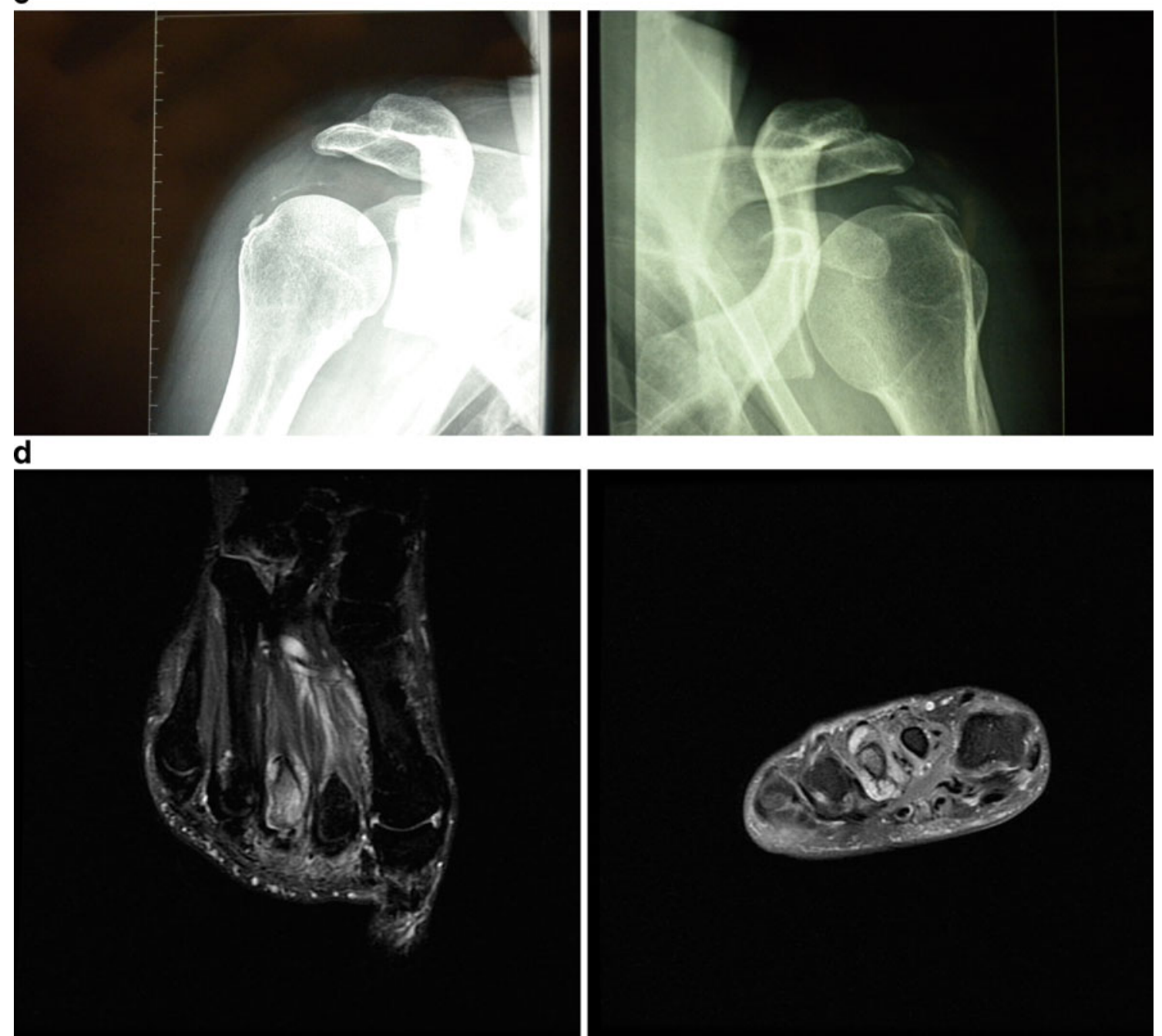

Fig. 2 a, Chikungunya infection, acute stage with edematous rash of the face. b, Chikungunya infection, chronic stage with swollen and stiff hands in a 55-year-old man who was infected 5 years earlier. c, Chikungunya infection, chronic stage with bilateral calcifications of

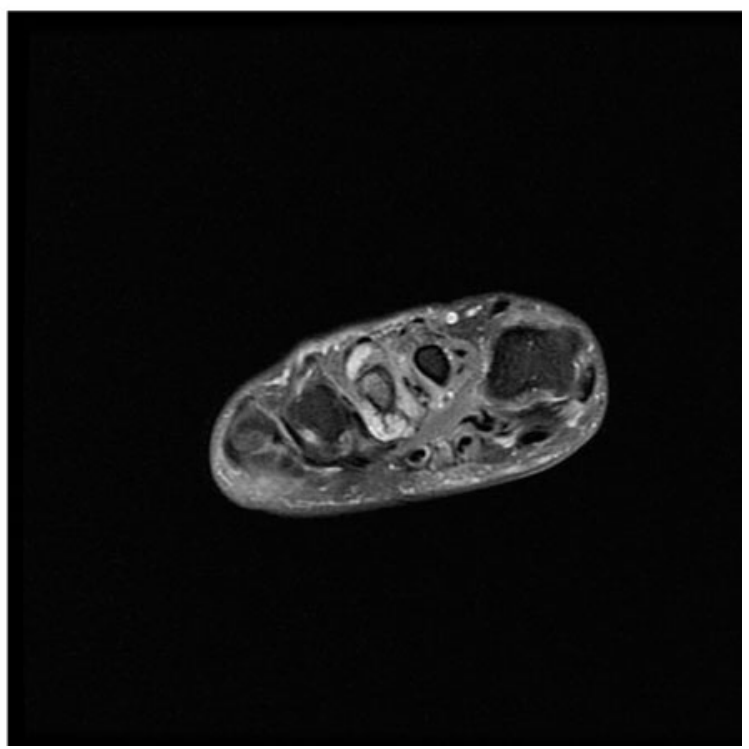

shoulder tendons in a 36-year-old woman who was infected 18 months previously. d, Chikungunya infection, chronic stage with inflammatory osteoarthritis of the second and third metatarsophalangeal joints on the left foot of a 43-year-old man who was infected 5 years earlier 
Table 2 Main clinical and biological differences between chikungunya and dengue fever

\begin{tabular}{lll}
\hline & Chikungunya & Dengue \\
\hline Clinical signs & & Common \\
Fever & Common & D5-D7 \\
Rash & D1-D4 & Common \\
Retroorbital pain & Rare & Rare \\
Arthralgia & Constant & Absent \\
Arthritis & Common, edematous & Common \\
Myalgia & Common & Absent \\
Tenosynovitis & Common & Common, D5-D7 \\
Hypotension & Possible & Common, D5-D7 \\
Minor bleeding & Rare & Possible fatigue for weeks \\
Outcome & Possible Raynaud syndrome, M2-M3 & \\
& Possible tenosynovitis, M2-M3 & Delayed and possibly deep \\
Biological changes & Common persistence of arthralgia for months to years & \\
Thrombocytopenia & & Early and mild
\end{tabular}

$D$ day, $M$ month

(Adapted from Simon et al. [1•].)

poor feeding, diffuse pain, distal joint edema, and miscellaneous skin alterations (petechiae, exanthema). Viral meningoencephalitis was the most common complication, with pathological MRI findings and poor outcomes (death, persistent disabilities).

\section{Chronic Stage}

After the short-lived improvement following the acute stage, the life of the patient recently infected with CHIKV can be impaired by early exacerbation, inflammatory relapses, long-lasting rheumatism, and a significant loss in the quality of life [9]. This deterioration is more frequent in patients over 40 years of age and/or with underlying diseases, notably rheumatic or traumatic diseases [41•]. High CHIKV viral loads in the acute stage are also associated with the persistence of symptoms [26••].

Within the first 3 months, most patients experience a rebound of general discomfort, inflammation in the joints and tendons, and an increased handicap in daily life [9]. Disabilities in the extremities are from a severe polyarthritis involving most distal joints and multiple hypertrophic tenosynovitides that are sometimes responsible for carpal or tarsal tunnel syndromes. Difficulties in walking and handling objects can induce frequent and/or prolonged sick leave. Transient vascular disorders (eg, Raynaud syndrome) are present in one patient in six at that period [9], possibly in relation with the concomitant presence of mixed cryoglobulinemia [25]. Ocular changes may also develop a few weeks after disease onset: anterior uveitis, retinitis, episcleritis, and optic neuritis, sometimes leading to blindness [42].

In Reunion Island, $80 \%$ to $90 \%$ of CHIKV-infected patients complained of persistent symptoms after the first 3 months $[41 \cdot, 43]$. Whether this prevalence of nonrecovery is similar in other epidemic countries remains to be determined. However, CHIKV-induced rheumatism is the most frequent manifestation of the chronic stage. It consists of three clinical components, singly or in combination: 1) distal polyarthritis or monoarthritis mildly improved with NSAIDs, 2) frequent tenosynovitides in the hands, wrists, or ankles, highly sensitive to short-term systemic corticotherapy, and 3) exacerbation of pain in previously injured joints and bones requiring painkillers [9]. Most of the time, chronic peripheral polyarthritis is not associated with significant biological changes or the appearance of autoantibodies [9], but mild mixed cryoglobulinemia is frequent [25]. First radiographs of the joints involved usually do not reveal significant changes, even in disabled patients. Relapses are common during the chronic stage, often triggered after exposure to cold. They include mild fever, asthenia, increased inflammation in previously involved joints, and occasionally in new joints. Intensification of symptomatic treatment is often necessary and additional localized treatments and physiotherapy can be of benefit to some patients.

The chronic stage can severely deteriorate the patient's quality of life for months. At 6 months, 97\% of French CHIKV-infected active adults complained of joint symptoms. They considered their fatigue as totally disabling in 
$4.6 \%$ of cases, very significant or significant in $42.8 \%$, and $37.8 \%$ described their mood as demoralized, weakened in $43.9 \%$, and normal in only $16.2 \%$ of cases [10]. Another study showed that, on average, only $56 \%$ of 199 CHIKVseropositive patients considered themselves as cured 17 months after infection, being significantly longer in older patients. Arthralgia, fatigue, depression, and sleep disorders were twice as common in these patients as among seronegative controls. However, the SF-12 questionnaire evaluated the physical component of the quality of life to be significantly lower among seropositive patients, but not the mental component [43]. De Andrade et al. [44•] evaluated the link between pain and quality of life in symptomatic patients, with a main impact on mood, work, and sleep.

Over time, unexpected delayed rheumatic complications have manifested years after acute CHIKV infection. A small percentage of patients with persistent arthralgia develop authentic inflammatory destructive rheumatisms [45-48]. The most common feature is a rheumatoid arthritis (RA)like polyarthritis following CHIKV infection. Criteria for this new entity are the presence of anti-CHIKV IgM and IgG antibodies, RA based on the 1987 American College of Rheumatology criteria, no other definite diagnosis for arthritis, and persistent arthritis symptoms from the onset of CHIKV infection to the RA diagnosis [47••].

Bouquillard and Combe [47••] reported a series of 21 cases of severe RA after CHIKV infection in Reunion Island. All patients were over 45 years old and most of them developed symmetric polyarthritis $(18 / 21)$ within an average of 10 months. The biological characteristics of the disease were significant systemic inflammation, the presence of rheumatoid factors and HLA DRB $1 * 04$ or 01 alleles in more than half the patients, and variability of antiCCP antibodies. Erosions and/or joint space narrowing were present on initial radiographs of hands and feet in half the patients. During a 2-year follow-up, most patients received systemic corticotherapy and were treated with disease-modifying antirheumatic drugs (DMARDs), mostly methotrexate (19/21) and tumor necrosis factor blockers (6/21). Other forms of joint destruction have been described, such as the severe relapse of previously well-controlled psoriatic arthritis, entesopathies, or periostitis [22, 45, 48]. MRI scans seem sensitive in detecting early changes in joints and tendons in CHIKV-induced rheumatism [46]. This could prompt intensification of therapy, including the use of DMARDs, if validated by further studies. To date, there is no evidence of viral reactivation in patients treated with such immunosuppressive drugs, but the benefits/risks balance must be cautiously and regularly evaluated for each patient. However, if indicated for a patient with post-CHIKV destructive arthritis, prescription of a first-line treatment with methotrexate should not be excluded just because of the viral origin of the rheumatism.

\section{Future Challenges}

\section{Scientific Progress}

Despite dramatic progress in our understanding of CHIKV infection, research must be continued into the pathogenesis of the long-lasting osteoarticular involvement. Studies should determine whether viral tissue sanctuaries exist in all chronic patients or not, and if any drug could help the immune system to eliminate CHIKV and improve patients. In parallel with the search for efficient antiviral drugs, a new strategy is being developed with human anti-CHIKV immunoglobulins. Specific immunoprophylaxis or immunotherapy could provide a rapid antiviral action for persons at risk of severe acute CHIKV disease, notably neonates born from viremic mothers [49•].

\section{Management of CHIKV-Infected Patients}

The most important challenge is to reduce chronic pain and handicap during the chronic stage of the disease. Neuropathic pains in persistently symptomatic patients require a specific pharmacological approach in regard to their association with a more aggressive clinical picture, and their impact on the quality of life [44•]. While awaiting future antiviral therapy for the chronic stage, an urgent need exists to study the efficacy and safety of corticotherapy and DMARDs, especially methotrexate, to establish a strategy for the early treatment of destructive arthritis.

\section{Globalization and Prevention}

The size and speed of international population movements and the progressive worldwide dissemination of the tiger mosquito increase the risk of new epidemic emergence of CHIKV. International collaboration in preparedness and response to CHIKV introduction in susceptible regions should limit this threat. Controls in epidemic countries can be improved by prompt vector control and the isolation of infective patients under bednets, efficient action that is still neglected. To date, there is no commercial vaccine against CHIKV. Because of the possible risk of viral persistence, live CHIKV vaccines have been abandoned in favor of new candidates such as consensus-based DNA vaccines [50].

\section{Conclusions}

Within less than a decade, CHIKV has become a new giant among arboviral diseases, next to dengue fever. This emergence is mostly the result of viral evolutionary convergence with Ae. albopictus. This epidemiological change has led to its global expansion, supported by the 
faster transport of CHIKV-viremic travelers to susceptible areas with Aedes spp activity. In epidemic countries, CHIKV is responsible for brutal outbreaks of febrile episodes, and, unlike dengue fever, for endemic chronic rheumatic disorders, the treatment and outcome of which remain uncertain. CHIKV raises modern challenges in science, patient care, and public health that should be promptly taken up to limit its current rapid spread and major consequences.

Acknowledgement The authors thank Dr. Geffroy for his technical support in MRI illustrations. This work was supported by the French Service de Santé des Armées.

Disclosure Conflicts of interest: F. Simon - none, E. Javelle - none, M. Oliver-none, I. Leparc-Goffart-none, and C. Marimoutounone.

Open Access This article is distributed under the terms of the Creative Commons Attribution Noncommercial License which permits any noncommercial use, distribution, and reproduction in any medium, provided the original author(s) and source are credited.

\section{References}

Papers of particular interest, published recently, have been highlighted as:

- Of importance

- Of major importance

1. - Simon F, Savini H, Parola P. Chikungunya: a paradigm of emergence and globalization of vector-borne diseases. Med Clin North Am. 2008;92:1323-43. This study details the miscellaneous clinical manifestations of chikungunya infection.

2. Pastorino B, Muyembe-Tamfum JJ, Bessaud M, et al. Epidemic resurgence of Chikungunya virus in democratic Republic of the Congo: identification of a new central African strain. J Med Virol. 2004;74:277-82.

3. Charrel RN, de Lamballerie X, Raoult D. Chikungunya outbreaks-the globalization of vectorborne diseases. N Engl J Med. 2007;356:769-71.

4. Josseran L, Paquet C, Zehgnoun A, et al. Chikungunya disease outbreak, Reunion Island. Emerg Infect Dis. 2006;12:1994-5.

5. Mavalankar D, Shastri P, Raman P. Chikungunya epidemic in India: a major public-health disaster. Lancet Infect Dis. 2007;7:306-7.

6. Hochedez P, Hausfater P, Jaureguiberry S, et al. Cases of chikungunya fever imported from the islands of the South West Indian Ocean to Paris, France. Euro Surveill. 2007; 12.

7. Update: chikungunya fever diagnosed among international travelers-United States, 2006. Morbidity and Mortality Weekly Report. 2007; 56: 276-7.

8. Rezza G, Nicoletti L, Angelini R, et al. Infection with chikungunya virus in Italy: an outbreak in a temperate region. Lancet. 2007;370:1840-6.

9. Simon F, Parola P, Grandadam M, et al. Chikungunya infection: an emerging rheumatism among travelers returned from Indian Ocean islands. Report of 47 cases. Medicine (Baltimore). 2007;86:123-37.
10. Queyriaux B, Simon F, Grandadam M, et al. Clinical burden of chikungunya virus infection. Lancet Infect Dis. 2008;8:2-3.

11. Borgherini G, Poubeau P, Staikowsky F, et al. Outbreak of chikungunya on Reunion Island: early clinical and laboratory features in 157 adult patients. Clin Infect Dis. 2007;44:1401-7.

12. Schuffenecker I, Iteman I, Michault A, et al. Genome microevolution of chikungunya viruses causing the Indian Ocean outbreak. PLoS Med. 2006;3:e263.

13. Volk SM, Chen R, Tsetsarkin KA, et al. Genome-scale phylogenetic analyses of chikungunya virus reveal independent emergences of recent epidemics and various evolutionary rates. J Virol. 2010;84:6497-504.

14. $\bullet$ Chevillon C, Briant L, Renaud F, Devaux C. The Chikungunya threat: an ecological and evolutionary perspective. Trends Microbiol. 2008;16:80-8. This review discusses up-to-date information on CHIKV genetics, ecology, and human infection.

15. Tsetsarkin KA, Vanlandingham DL, McGee CE, Higgs S. A single mutation in chikungunya virus affects vector specificity and epidemic potential. PLoS Pathog. 2007;3:e201.

16. Vazeille M, Moutailler S, Coudrier D, et al. Two Chikungunya isolates from the outbreak of La Reunion (Indian Ocean) exhibit different patterns of infection in the mosquito, Aedes albopictus. PLoS ONE. 2007;2:e1168.

17. Santhosh SR, Dash PK, Parida MM, et al. Comparative full genome analysis revealed E1: A226V shift in 2007 Indian Chikungunya virus isolates. Virus Res. 2008;135:36-41.

18. de Lamballerie X, Leroy E, Charrel RN, et al. Chikungunya virus adapts to tiger mosquito via evolutionary convergence: a sign of things to come? Virol J. 2008;5:33.

19. Couderc T, Chretien F, Schilte C, et al. A mouse model for Chikungunya: young age and inefficient type-I interferon signaling are risk factors for severe disease. PLoS Pathog. 2008;4:e29.

20. Couderc T, Lecuit M. Focus on Chikungunya pathophysiology in human and animal models. Microbes Infect. 2009;11:1197205.

21. • Labadie K, Larcher T, Joubert C, et al. Chikungunya disease in nonhuman primates involves long-term viral persistence in macrophages. J Clin Invest. 2010;120:894-906. This study describes a new model for CHIKV infection in adult immunocompetent cynomolgus macaques. CHIKV infection in these animals recapitulates the viral, clinical, and pathological features reported in human cases.

22. Jaffar-Bandjee MC, Das T, Hoarau JJ, et al. Chikungunya virus takes centre stage in virally induced arthritis: possible cellular and molecular mechanisms to pathogenesis. Microbes Infect. 2009;11:1206-18.

23. Sourisseau M, Schilte C, Casartelli N, et al. Characterization of reemerging chikungunya virus. PLoS Pathog. 2007;3:e89.

24. Ozden S, Huerre M, Riviere JP, et al. Human muscle satellite cells as targets of chikungunya virus infection. PLoS ONE. 2007;2: e527.

25. Oliver M, Grandadam M, Marimoutou C, et al. Persisting mixed cryoglobulinemia in Chikungunya infection. PLoS Negl Trop Dis. 2009;3:e374.

26. •- Hoarau JJ, Jaffar Bandjee MC, Krejbich Trotot P, et al. Persistent chronic inflammation and infection by Chikungunya arthritogenic alphavirus in spite of a robust host immune response. J Immunol. 2010;184:5914-27. This prospective cohort study highlights cellular and molecular events that may lead to chronic arthralgia/arthritis.

27. Sergon K, Yahaya AA, Brown J, et al. Seroprevalence of Chikungunya virus infection on Grande Comore Island, union of the Comoros, 2005. Am J Trop Med Hyg. 2007;76:1189-93.

28. Krishnamoorthy K, Harichandrakumar KT, Krishna Kumari A, Das LK. Burden of chikungunya in India: estimates of disability 
adjusted life years (DALY) lost in 2006 epidemic. J Vector Borne Dis. 2009;46:26-35.

29. Panning M, Hess M, Fischer W, et al. Performance of the RealStar Chikungunya virus real-time reverse transcription-PCR kit. J Clin Microbiol. 2009;47:3014-6.

30. • Niedrig M, Zeller H, Schuffenecker I, et al. International diagnostic accuracy study for the serological detection of chikungunya virus infection. Clin Microbiol Infect. 2009;15:880-4. This study investigates the quality of different serological tests.

31. World Health Organization. Guidelines on clinical management of chikungunya fever. 2008.

32. De Lamballerie X, Boisson V, Reynier JC, et al. On chikungunya acute infection and chloroquine treatment. Vector Borne Zoonotic Dis. 2008;8:837-9.

33. Hochedez P, Jaureguiberry S, Debruyne M, et al. Chikungunya infection in travelers. Emerg Infect Dis. 2006;12:1565-7.

34. Renault P, Solet JL, Sissoko D, et al. A major epidemic of chikungunya virus infection on Reunion Island, France, 20052006. Am J Trop Med Hyg. 2007;77:727-31.

35. Tournebize P, Charlin C, Lagrange M. Neurological manifestations in Chikungunya: about 23 cases collected in Reunion Island. Rev Neurol (Paris). 2009;165:48-51.

36. Simon F, Paule P, Oliver M. Chikungunya virus-induced myopericarditis: toward an increase of dilated cardiomyopathy in countries with epidemics? Am J Trop Med Hyg. 2008;78:212-3.

37. Economopoulou A, Dominguez M, Helynck B, et al. Atypical Chikungunya virus infections: clinical manifestations, mortality and risk factors for severe disease during the 2005-2006 outbreak on Reunion. Epidemiol Infect. 2009;137:534-41.

38. Ernould S, Walters H, Alessandri JL, et al. Chikungunya in paediatrics: epidemic of 2005-2006 in Saint-Denis, Reunion Island. Arch Pediatr. 2008;15:253-62.

39. Gerardin P, Barau G, Michault A, et al. Multidisciplinary prospective study of mother-to-child chikungunya virus infections on the island of La Reunion. PLoS Med. 2008;5:e60.

40. Manimunda SP, Mavalankar D, Bandyopadhyay T, Sugunan AP. Chikungunya epidemic-related mortality. Epidemiol Infect. 2010:1-3.
41. - Sissoko D, Malvy D, Ezzedine K, et al.: Post-epidemic Chikungunya disease on Reunion Island: course of rheumatic manifestations and associated factors over a 15-month period. PLoS Negl Trop Dis. 2009;3:e389. This study investigates potential risk factors associated with persistent rheumatic manifestations at the late stage of the CHIKV infection.

42. Mahendradas P, Ranganna SK, Shetty R, et al. Ocular manifestations associated with chikungunya. Ophthalmology. 2008;115:287-91.

43. Soumahoro MK, Gerardin P, Boelle PY, et al. Impact of Chikungunya virus infection on health status and quality of life: a retrospective cohort study. PLoS ONE. 2009;4:e7800.

44. - de Andrade DC, Jean S, Clavelou P, et al. Chronic pain associated with the Chikungunya Fever: long lasting burden of an acute illness. BMC Infect Dis. 2010;10:31. This cross-sectional study evaluates chikungunya infection's impact on quality of life.

45. Malvy D, Ezzedine K, Mamani-Matsuda M, et al. Destructive arthritis in a patient with chikungunya virus infection with persistent specific IgM antibodies. BMC Infect Dis. 2009;9:200.

46. Manimunda SP, Vijayachari P, Uppoor R, et al.: Clinical progression of chikungunya fever during acute and chronic arthritic stages and the changes in joint morphology as revealed by imaging. Trans R Soc Trop Med Hyg. 2010;104:392-9.

47. •• Bouquillard E, Combe B. A report of 21 cases of rheumatoid arthritis following Chikungunya fever. A mean follow-up of 2 years. Joint Bone Spine. 2009;76:654-7. This paper defines a new clinical entity: rheumatoid arthritis following chikungunya infection.

48. Chopra A, Anuradha V, Lagoo-Joshi V, et al. Chikungunya virus aches and pains: an emerging challenge. Arthritis Rheum. 2008;58:2921-2.

49. • Couderc T, Khandoudi N, Grandadam M, et al. Prophylaxis and therapy for Chikungunya virus infection. J Infect Dis. 2009;200:516-23. This study reports the in vitro effectiveness of human anti-chikungunya immunoglobulins, the importance of which lies in the current lack of effective antiviral drugs.

50. Muthumani K, Lankaraman KM, Laddy DJ, et al. Immunogenicity of novel consensus-based DNA vaccines against Chikungunya virus. Vaccine. 2008;26:5128-34. 